\title{
TREATMENT OF PARAPLEGIC SPASTICITY BY MUSCLE TRANSPLANTATION
}

\author{
MARIAN WEISS, M.D. \\ Boleslaw Bielicki, Warsaw, Poland
}

SPASTICITY resulting from trauma or from a variety of diseases of the central nervous system is a difficult problem for a rehabilitation clinic. Increased muscular tension, usually prevailing in the flexor groups, leads to secondary irreversible morphological changes in the hip, knee and ankle joints. An uneven distribution of the pressure-force on the joint cartilage causes its destruction, and capsule contractures and partial dislocations of various degrees may occur.

The unavoidable position of the patient on his side causes trophic changes in the skin and is an obstacle to regular care, encouraging the occurrence of bedsores. It is frequently difficult to prevent such complications in the presence of severe spasticity.

It becomes impossible to obtain adequate functional efficiency even for such minimum demands as adaptation to use a wheelchair. Every active movement causes increased muscle tension in the paretic muscles even amounting to 'mass reflexes'.

Study of the material of the School of Medicine Rehabilitation Institute and of recent publications demonstrates that a diminution or elimination of increased muscle tension allows greater functional efficiency. In severe cases of spastic paraplegia, the aim should be that the patient should adopt a stable sitting position as this is the position he will require to retain for the rest of his life.

Diminution of spasticity often permits braces to be used, allows the patient to be upright and to walk unassisted for at least a short distance. This will fundamentally alter the patient's psychological and social attitudes.

Diminution of spasticity can be obtained:

I. by means of exclusion of various links of the reflex arc:

(a) by surgery, by dividing appropriate components of the reflex arc;

(b) chemically, by a temporary block of nervous conduction (phenol, alcohol);

2. by diminishing the contraction potential of the muscle by elongation of the spastic muscle tendon.

Clinical Studies on the Surgery of Spastic Paraplegia. In patients with lower extremity paralysis transferred to the Rehabilitation Institute from various centres throughout Poland, cases with spastic paraplegia with fixed contractures were frequently encountered. Lower extremity flexion-contractures of various degrees were common and the greatest obstacle to attempts at standing were presented by clubfoot deformity. In patients in whom voluntary action of trunk and shoulder muscles was preserved (in the young and relatively efficient person), it was worth while starting attempts at a mechanical reconstruction of the body axis which would ensure a vertical position. Since the clubfoot usually interfered with these plans, elongation of the Achilles tendon was performed. The operation usually allowed the patient to be upright so that he could be taught to walk un- 
assisted with the help of appropriate technical aids. There was a reduction of spasticity both in the feet and in the knees. It sometimes happened that the attachment of the extremity to a straight plaster rail by bandages became possible only
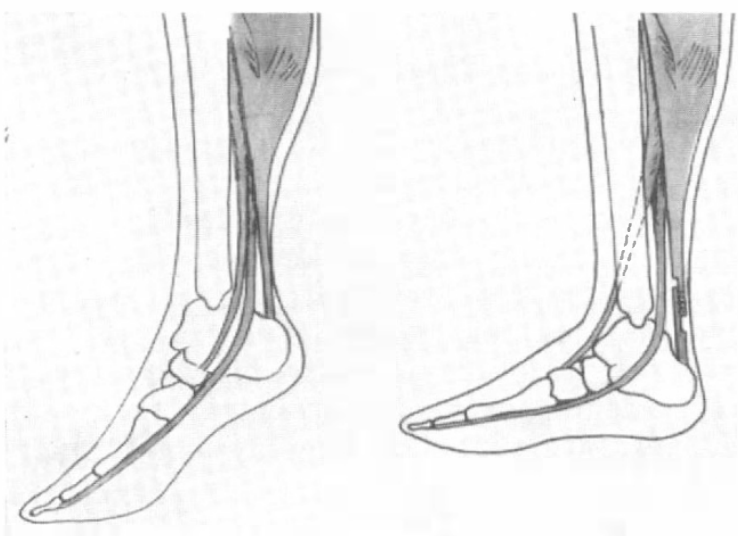

FIG. I

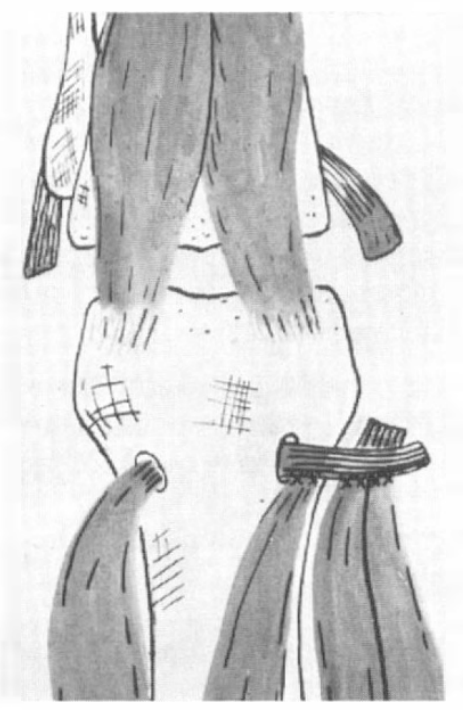

FIG. 2

after operative treatment. Previously, the tension in the group of flexors prevented any passive extension of the knee joint. Subsequent observation indicated that, because of an inconsistent corrective positioning of the extremities, the clubfoot reappeared.

It was therefore essential to create a counterbalance for the contracted calf triceps and for this the tibialis posterior muscle was transplanted through the interosseous membrane to the base of the third metatarsal bone (fig. I). This 
muscle, as the synergist of the calf triceps, was in a spastic state, which is usually demonstrated by the talipes adductor position of the foot. When transplanted under appropriate tension to the dorsum of the foot, it probably does not lose any of its pathological tension, and at the same time the elongated Achilles tendon causes a shortening of the belly of the calf triceps and thereby reduces its spasticity. The result of this operation was long lasting, particularly if the patient spent a few hours each day in the vertical position, and when a corrective position was maintained in bed. Diminution or abolition of ankle clonus also occurred, and there was a decrease of spastic tension in the knee flexors. This permitted the application of orthopaedic apparatus and training in walking.

In more severe cases it was decided to carry out the second stage of the operation, i.e. transplantation of the hamstrings into the distal part of the femur. Further clinical observations revealed that it was possible to carry out simultaneous operations on the feet and on the knee muscles (fig. 2).

Good functional results in cases of paraplegia led to the application of similar surgical treatment in cases of spastic paraplegia of cerebral origin, i.e. in hemiplegia. In such patients, however, it was necessary to grade the operation according to the degree of spasticity and to the extent of contracture. Thus in the less severe cases, instead of elongation of the Achilles tendon, the operation of Vulpius was performed, or a disinsertion of the beads of the gastrocnemius by Silverskjöld's technique. Similarly, the tibialis anterior was transplanted to the dorsum of the foot, preserving voluntary action.

Criteria for the Assessment of Treatment. Forty patients with traumatic paraplegia were studied. Patients with hemiparesis were studied separately but the same criteria for the evaluation of the surgical results were used.

I. Functional Examination. In addition to regular clinical examinations attention was principally directed to the functional condition of the patient, such as gait, performance of routine daily activities and self-care. The functional status was tested and points were awarded depending on the degree of efficiency. As the degree of contracture appeared to be important, the morphological evaluation of contractures was also included.

Functional evaluation is applicable exclusively to patients with second and third degrees of spasticity. In the first degree of spasticity, when the patient was fully efficient, only the gait was taken into consideration.

2. Film. In order to obtain an objective assessment of the results of treatment the patient's gait was recorded on film.

3. EMG Examinations. These were made by the three-channel technique, using surface electrodes. The tibialis anterior, gastrocnemius and the quadriceps of the hip were studied during voluntary and passive movements, during stimulation of tendon reflexes and when walking. The effects of operative treatment were studied.

4. Social Results. In considering the social results of treatment emphasis was laid on his degree of independance and on the eventual resumption of work.

5. Subjective Assessment. The subjective evaluation of treatment is essential, although it is not always comparable with objective evaluation both as regards positive and negative aspects. This discrepancy between objective and subjective assessment was encountered principally in patients with serious psychological abnormalities. Most of these were hemiparetics. 
Before the suggested operation, attempts were made to obtain the patient's acceptance of the plan by explaining the nature of the operation to him. He was encouraged to have an active attitude to motor exercises prior to and after the operation. Complete recovery of function was never promised.

Results. Operations were performed on 40 patients with paralysis or spastic spinal paraplegia (Table I). Functionally, the efficiency was poor and 36 were

TABLE I

\begin{tabular}{|c|c|c|c|c|c|} 
& \multicolumn{5}{c}{ Etiology-Age } \\
\cline { 3 - 6 } Age & Trauma & $\begin{array}{c}\text { Transverse } \\
\text { myelitis }\end{array}$ & Tumor & Other & Total \\
\hline I0-20 & 4 & 6 & - & 3 & I3 \\
2 I-40 & I & 4 & I & 5 & 24 \\
4 I-50 & I & - & - & - & I \\
5I-60 & - & I & - & - & I \\
$60<$ & - & I & - & - & I \\
\hline Total & I9 & I2 & I & 8 & 40 \\
\hline
\end{tabular}

TABLE II

Division of Clinical Material depending on the Degree of Spasticity

\begin{tabular}{|c|c|c|c|c|c|}
\hline & $\begin{array}{c}\text { Degree of } \\
\text { spastic }\end{array}$ & Trauma & $\begin{array}{c}\text { Transverse } \\
\text { myelitis }\end{array}$ & Tumor & Other \\
\hline-4 & I & - & - & - & - \\
\hline 36 & II & 2 & 2 & - & - \\
\hline 40 & Total & I9 & I2 & I & 8 \\
\hline
\end{tabular}

unable to assume the vertical position and were unable to walk. Six patients could not leave their beds and, because of strong spasticity, were unable to use a wheelchair (Table II). No voluntary action of the lower extremity was perceptible in any of these patients, except for a trace of voluntary action in the quadriceps femoris in five. The remainder, i.e. 25 patients, showed complete spastic paralysis of the flexion type. Four patients were able to walk with the aid of parallel bars but their gait was inefficient and spastic, of the type known as 'stork' gait (Table III).

As regards the degree of spasticity, 36 patients were in group 3 and four in group 2.

An improvement in gait was obtained in four cases and 32 patients were able to walk with elbow crutches and braces. In the four cases indicated in the table as a 'bad' result, a reduction in spastic tension was obtained, enabling them to use a wheelchair. However, their poor general condition and in particular the weak upper extremities and shoulder muscles did not allow them to be upright (Table IV). 
As mentioned above, an EMG tracing of voluntary action in the quadriceps femoris was seen in five patients. These patients had had operations on the feet, and also transplantation of the leg flexors to the femur, which resulted in much better voluntary action of the quadriceps femoris. The power of these muscles was

TABLE III

Action of the Antagonists

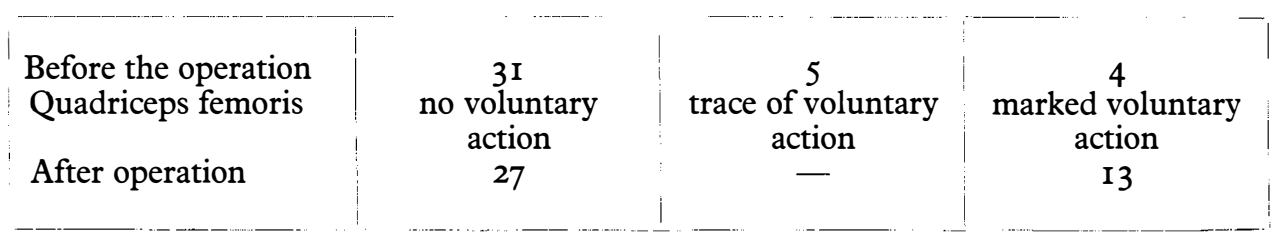

TABLE IV

Functional Status Before and After Surgery

\begin{tabular}{|l|r|r|}
\hline Function & Before & After \\
\hline Bed . & 6 & - \\
Wheel chair independent & 34 & 40 \\
Independent walk . & 4 & 36 \\
\hline
\end{tabular}

sufficient for stabilisation of the knee joints during walking. In four patients (in whom transplantation of hamstrings to the femur was performed) voluntary action of the quadriceps muscles, imperceptible before the operation, was apparent after it. Gradual strengthening of these muscles resulted in an increased stability of the knee joints.

In summary, 29 patients could maintain a vertical position, with braces and crutches. In four patients the gait was considerably improved. Seven patients were able to sit in and use a wheelchair. It is obvious that the fundamental position, which will become the position for their lifetime, is the sitting position, but walking unassisted for even a limited distance is of practical and psychological value.

The gait obtained after operative treatment varied from taking a few steps indoors to efficient walking, enabling ascent of stairs (six cases). But this depends on many factors, such as the level of the spinal injury, the extent of paralysis, the general condition of the patient, strength of the upper extremities, weight of the body, obesity and age.

\section{DISCUSSION}

Our conclusion is that improvement of general functional efficiency was obtained in every patient. Even where it was not possible to have the patient upright, greater motor efficiency was obtained, enabling self-care and locomotion in a wheelchair. Decrease in spasticity was achieved in every case. Recovery of walking function was obtained in 29 cases, a result which would have been impossible without operation.

EMG examinations confirmed the clinical observations (this will be the 
subject of a subsequent paper). They showed that reflex activity increases; there is also some improvement in the co-ordination of the agonist-antagonist action.

In two cases simultaneous extensive tenotomy of all the flexors was made, i.e. of the hip-joint flexors and adductors, and of the flexors of the joints of the knee and foot. It was then possible passively to stretch the remaining elements, the skin, the vessels, the nerves and the joint capsules to a degree permitted by deformation of the joint surfaces. This was not standard procedure and was not included in the specifications of our clinical material; none the less, it enabled patients to assume the sitting position and facilitated their care. The indication for such an extensive operation was fixed, maximal flexion-adduction contractures in the hip, knee and ankle joints.

In mild degrees of spasticity an operation on the foot was enough, with subsequent application of appropriate braces, but in more severe cases transplantation of the knee flexors to the femur was performed. The action of these muscles is at first relatively diminished by the reduction of their tension but later, after adaptation, these muscles help in extension of the hip joint and stabilise the knee by drawing the hip backwards. Transplantation of hamstrings to the femur was usually combined with the peripheral transplantation of the tibial tuberosity, thus providing better conditions for the function of the quadriceps of the thigh.

In one case it was necessary to perform a second operation to adduct the tibial tuberosity previously peripherally transplanted, as the muscle tension caused hyperflexion of the knee.

Functional improvement was seen in all the operated cases, and 29 were able to assume a vertical position and to move unassisted over a limited distance.

Patients, previously bedridden, were trained after operation for the sitting position, and to be as independent as possible for all ordinary activities.

\section{CONCLUSIONS}

The clinical observations together with the electromyographic analysis ${ }^{\star}$ of the material outlined in this research support the view that there is a fundamental improvement in the patients treated by operation.

I. The indirect setting up of the foot, obtained by elongation of the Achilles tendon and the transplantation of the tibialis posterior to the dorsum of the foot, creates favourable conditions for developing a compensatory sterotype of the foot. This also has an influence on proprioception.

2. The operations performed cause a diminution in the increased tension in the flexor muscles of the large joints of the lower extremities.

3. The diminution of this spasticity causes a decrease of the proprioceptive inhibition in the anti-gravity muscles.

4. Transplantation of the hamstrings causes a new dynamic-static system in the knee joint, improving anti-gravity action.

5. The operative procedures advised cause a decrease of inhibition in the groups of antagonists. Stress is therefore placed on the appearance of voluntary function of the quadriceps of the hip after transplantation of the knee joint flexors; this was observed in four cases of spastic paraplegia.

6. The surgical treatment here discussed enables the patient to obtain better motor efficiency when compared to their pre-operative condition.

$\star$ This will be the subject of a subsequent paper. 